\title{
Gary Ferguson, Catherine Hampton, a cura di, (Re)Inventing the Past
}

\section{Filippo Fonio}

\section{(2) OpenEdition}

1 Journals

\section{Edizione digitale}

URL: http://journals.openedition.org/studifrancesi/34478

DOI: 10.4000/studifrancesi.34478

ISSN: 2421-5856

\section{Editore}

Rosenberg \& Sellier

\section{Edizione cartacea}

Data di pubblicazione: 1 novembre 2005

Paginazione: 398-399

ISSN: 0039-2944

\section{Notizia bibliografica digitale}

Filippo Fonio, «Gary Ferguson, Catherine Hampton, a cura di, (Re)Inventing the Past», Studi Francesi

[Online], 146 (XLIX | II) | 2005, online dal 30 novembre 2015, consultato il 20 avril 2021. URL: http:// journals.openedition.org/studifrancesi/34478; DOI: https://doi.org/10.4000/studifrancesi.34478

Questo documento è stato generato automaticamente il 20 avril 2021.

\section{(c) $($ i) $(9)$}

Studi Francesi è distribuita con Licenza Creative Commons Attribuzione - Non commerciale - Non opere derivate 4.0 Internazionale. 


\title{
Gary Ferguson, Catherine Hampton, a cura di, (Re)Inventing the Past
}

\author{
Filippo Fonio
}

\section{NOTIZIA}

GARY FERGUSON, CATHERINE HAMPTON, a cura di, (Re)Inventing the Past. Essays on French Early Modern Culture, Literature and Thought in Honour of Ann Moss, University of Durham («Durham Modern Languages Series»), 2003, pp. 331.

1 La miscellanea in onore di Ann Moss raccoglie contributi dedicati a questioni Cinque e Secentesche delle quali anche la studiosa si è occupata. Fra l'altro, la fortuna di Ovidio nel Rinascimento, Ronsard e i poeti della Pléiade, Montaigne, i repertori di loci communes, l'organizzazione delle categorie concettuali nel passaggio dal latino ai volgari e in seguito all'invenzione della stampa.

2 Dopo una bibliografia degli scritti della studiosa, il saggio di Ann Moss che apre il volume, Languages of the Renaissance (pp. 19-33), individua le linee della temperie culturale del secondo quarto del XVI secolo, periodo decisivo per la diffusione del latino degli Umanisti a sfavore del latino tardo-medievale. Osservando in particolare quanto concerne il linguaggio dell'argomentazione, nella duplice prospettiva delle dispute teologiche e accademiche - del resto spesso strettamente connesse - la studiosa porta alcuni esempi significativi in tal senso, fra i quali i commenti di Paolo Cortesi e John Mair alle Sententiae di Pietro Lombardo, l'apporto di Melantone e in generale della Riforma, il peculiare bilinguismo di Montaigne. I repertori di loci communes, fra i capisaldi dell'educazione umanistica, sono al centro non soltanto di un ripensamento degli strumenti dell'argomentazione, ma anche dell'affermarsi di una nozione di verità non dogmatica, che la nuova applicazione del sistema delle auctoritates porta con sé.

3 La maniera in cui tali repertori funzionano come materiali preparatori a diversi livelli è esemplificata nei contributi dedicati a Rabelais. TERENCE CAVE, Thinking with Commonplaces: the example of Rabelais, pp. 35-49, mostra come il sistema della cultura del 
Rinascimento agisca in maniera particolarmente evidente in quello che lo studioso definisce «fictional equivalent» di un libro di loci communes (Ivi, p. 40). L'importanza dei repertori per l'intellettuale del Cinquecento, e il legame della pratica compilatoria con l'avvento della stampa («the compilation of topoi becomes [...] a core activity of human cultural evolution, simultaneously preserving, recycling, extending and reassessing a corpus of potentially usable data», Ivi, pp. 36-37) vengono illustrati da Cave in riferimento a Rabelais, con esempi tratti dal Tiers Livre (la retorica di Epistemon) e dal Quart Livre (l'episodio delle parole congelate). Come invece Rabelais faccia uso del topos della curiositas e dei loci a esso collegati (cfr. in part. Pantagruel, IX, XIII) è oggetto del saggio di NEIL KENNY, Plautus, Panurge and 'les aventures des gens curieux', pp. 51-71. Kenny prende le mosse dagli studi di Gérard Defaux, per il quale i passi in questione sarebbero da ricondurre alla curiositas di Odisseo (nel senso di discendi cupiditas), e di Alfred Foulet, che legge curiaux da curieux tradito (da ricollegare a cura ma pure a curia). L'analisi si focalizza sulla componente intertestuale degli episodi del primo incontro fra Pantagruel e Panurge, e dell'avventura di Panurge fra i Turchi. Sulla scorta delle citazioni riportate dal Dictionarium di Robert Estienne (1531), Kenny individua una serie di influenze che con ogni probabilità l'autore del Pantagruel conosce, fra cui un passo dell'Aulularia di Plauto e uno da Varrone. Di nuovo a livello di intertestualità agisce il Corpus Priapceorum negli Essais di Montaigne come nelle opere di Rabelais (RICHARD COOPER, 'De la dignité des braguettes': Rabelais and Montaigne as readers of the «Priapea», pp. 73-104). La raccolta dei Priapea in particolare viene impiegata in diverse occasioni da Rabelais (cfr. ad ex. Cinquiesme livre, XLIII, o Prologo al Quart Livre), letta attraverso gli stilemi dell'eroicomico. Un esempio significativo è l'epiteto trismegiste mutuato dal rituale ermetico, mediante il quale Rabelais fa riferimento alla Dive Bouteille, ma pure, in una revisione del Pantagruel risalente al 1542, alla braguette di Panurge.

ALISON SAUNDERS si occupa delle raccolte di emblemi edite in Francia attorno alla metà del Cinquecento ('Disoit jadis le bon poete Homere, que Juppiter...': classical reworkings in early French vernacular emblem -books, pp. 105-123). A partire dagli anni Trenta del secolo, con la pubblicazione della raccolta di Andrea Alciato (al 1536 risale la traduzione francese curata da Jean Le Fevre), si assiste in Francia a una certa fortuna del genere, in un primo tempo sotto forma di opere in francese (Guillaume de La Perrière, Le Theatre des bons engins; Gilles Corrozet, Hecatomgraphie, 1540), che seguono la tradizione vernacola della poesia moraleggiante. A ciò fa seguito un ritorno al latino e a una più decisa impronta classica, con l'eccezione del Premier livre des emblems di Guillaume Guéroult (1550).

5 GARY FERGUSON, nel contributo Reviving Epic in Renaissance France: Ronsard, Jamyn, and other Homers, pp. 125-152, osserva la maniera in cui la Francia del Cinquecento si misura con il modello omerico, in particolare per ciò che concerne l'Iliade. Ferguson prende in considerazione i testi-soglia legati ad alcune iniziative editoriali collocabili fra epica e roman, nella fattispecie la Franciade di Ronsard, la traduzione francese dell'Iliade iniziata da Salel e portata a compimento da Jamyn, segretario di Ronsard, e quella dell'Amadis de Gaule. L'analisi evidenzia diverse questioni cardine per la cultura francese del Rinascimento, fra cui il dibattito sul valore artistico della traduzione, la riflessione sui generi poetici e la possibilità di dare vita a un epos di ispirazione autoctona. Sempre a problemi di teoria letteraria è legato lo studio di MICHEL MAGNIEN, Une poétique française inconnue: le de Arte poetica liber de Jean de La Fosse (1545), pp. 153-175. L'importanza del trattato in questione, opera dell'insegnante di un collegio della capitale, risiede nel 
fatto che si tratta di una delle prime poetiche francesi che mostrano un'influenza diretta della Poetica di Aristotele, fra l'altro nel valore attribuito alla prospettiva antiquaria e nell'interesse pressoché esclusivo per la poesia drammatica. L'ars poetica di Jean de La Fosse inquadra tuttavia tale impronta aristotelica in una teoria letteraria che trova come fondamento, anziché la mimesis aristotelica, il furor del poeta ispirato dagli dei.

6 Due contributi sono dedicati alla poesia di Remy Belleau. Nel primo, Jacopo Sannazaro's Arcadia and Remy Belleau's Bergerie (pp. 177-194), JEAN BRAYBROOK individua analogie e differenze nei due prosimetri di ambientazione bucolica, a partire proprio dal milieu culturale degli autori e dalla maniera in cui le vicende politico-sociali della seconda metà del Cinquecento vengono rispecchiate in particolare nella Bergerie di Belleau. $\mathrm{Al}$ solipsismo e alla melanconia del modello si sostituiscono la passione e la gioia d'amore che pervadono la Joinville di Belleau, il ruolo attivo delle figure femminili, il senso di comunità che ne traspare. Il secondo, FRANçOIS RIGOLOT, Métamorphoses de l'escargot: morale de l'expression chez Remy Belleau et Francis Ponge (pp. 195-214) è invece incentrato sulla maniera in cui due letterati per molte ragioni lontanissimi fra loro, Remy Belleau e Francis Ponge appunto, trattano un medesimo motivo, quello della lumaca. Se l'inventio può dirsi una infatti, dispositio ed elocutio differiscono in accordo con il progetto scientifico-mitologico dell'hymne-blason L'Escargot di Belleau da un lato, con il rifiuto del meraviglioso e l'impronta à la moraliste del Ponge di Escargots dall'altro.

7 Anche a Pierre de Ronsard sono dedicati due contributi. MALCOLM QUAINTON, in Horses for Courses: Ronsard's windy rhetoric (pp. 215-229), ricostruisce il tessuto retorico del componimento L'Ombre du Cheval (1568). Oscillando fra divertissement ed erudizione umanistica, poetica ed esercizio della fantaisie e della memoria in quanto evocazione in absentia, imitazione e inventio, L'Ombre du Cheval è caratterizzata da una fitta serie di richiami alla Classicità come al resto della lirica ronsardiana, in particolare a La Lyre e a l'Elegie du Verre. La figura di Narciso e le sue valenze letterarie e storiche in Ronsard, Les Amours, sonetti CCXXIV-CCXXVI, sono invece oggetto del saggio di JOHN O'BRIEN, Réflexivité et Réflectivité: le Narcisse ronsardien, pp. 231-244. L'autore parte dalla nozione di réflexivité elaborata da Paul Zumthor, osservando come «Ronsard [...] fait de la situation narcissique le moment privilégié de la réflexivité littéraire par la création et l'emploi d'un lexique que la véhicule» (Ivi, p. 234).

8 PHILIP FORD ricostruisce i contorni della figura di Camille de Morel, figlia di Jean de Morel (Camille de Morel: female erudition in the French Renaissance, pp. 245-259), nel quadro dell'educazione femminile nel Rinascimento. Lo studioso si sofferma in particolare su un'ode del padre e su un epigramma di Joachim Du Bellay, mostrando la maniera in cui la giovane poetessa era celebrata dagli intellettuali dell'epoca, non soltanto parigini. La seconda parte dell'intervento è dedicata al Tumulus per la scomparsa di Jean de Morel (1583), contenente diversi componimenti della figlia, e mette in evidenza come la lirica di Camille non si discosti dai modelli educativi conservatori proposti per le giovani dell'epoca.

9 Il complesso legame fra castità e desiderio, fra amore platonico e dinamiche di conservazione della società patriarcale sono oggetto del contributo di CATHY HAMPTON, Re-Negotiating Past Contracts: love and duty in the Querelle des Amyes and in the Renaissance 'roman sentimental', pp. 261-280. La studiosa prende in considerazione alcuni testi della Querelle des Amyes, in particolare La Parfaicte Amye di Antoine Héroët, l'Amye de Court di Betrand de la Borderie e il Nouvel Amour di Almanque Papillon, confrontandoli con 
alcuni esempi di roman sentimental, genere la cui diffusione in Francia si situa attorno alla fine del Cinquecento.

10 GILLIAN JONDORF si occupa dei caratteri della 'romanità' (con attenzione precipua alla constantia) in alcune tragedie Cinque e secentesche (Roman and Foreign in some French Tragedies, pp. 281-298). L'analisi è incentrata su alcune eroine tragiche, paradossalmente regine straniere, in realtà depositarie degli autentici valori del genus italicum: Cleopatra (Etienne Jodelle, Cléopâtre captive; Robert Garnier, Marc Antoine), Didone (Jodelle, Didon se sacrifiant), Sofonisba (Pierre Corneille, Sophonisbe; Jean de Mairet, La Sophonisbe), Berenice (Jean Racine, Bérénice).

11 INGRID A.R. DE SMET, infine, individua le caratteristiche del voyage galant, partendo dalla Relation d'un voyage en Limousin di Jean de La Fontaine, e cercando in alcuni modelli gli elementi del genere (From Brindisi to the Limousin: treading the path of the 'voyage galant', pp. 299-318) 